
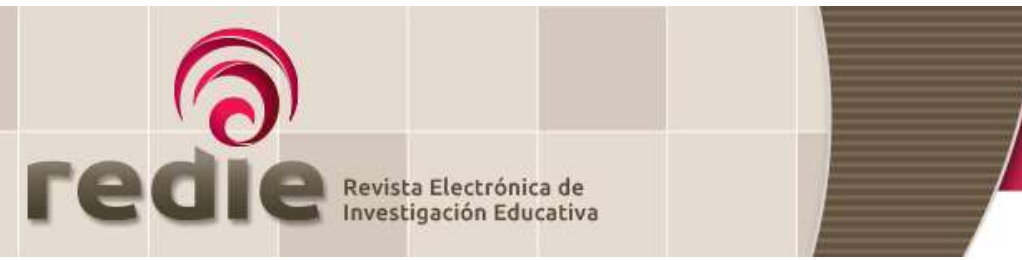

\title{
Intercambios comunicativos y práctica pedagógica en el aula de los docentes en formación ${ }^{1}$
}

\section{Communicative Exchanges and Classroom Teaching Practices of Trainee Teachers}

Marco Antonio Villalta Paucar (1) https://orcid.org/0000-0002-7553-925X
Sergio Martinic Valencia (2) https://orcid.org/0000-0003-2587-2414

(1) Universidad de Santiago de Chile

(2) Pontificia Universidad Católica de Chile

(Recibido: 11 de junio de 2018; Aceptado para su publicación: 1 de octubre de 2018)

Cómo citar: Villalta, M. A. y Martinic, S. (2020). Intercambios comunicativos y práctica pedagógica en el aula de los docentes en formación. Revista Electrónica de Investigación Educativa, 22, e21, 1-16. https://doi.ora/10.24320/redie.2020.22.e21.2513

\section{Resumen}

Las prácticas profesionales en la formación inicial de los docentes constituyen una experiencia de saber situado de la enseñanza. En este artículo se hace un análisis de las clases de estudiantes en el marco de sus prácticas profesionales; se observó a seis estudiantes de Pedagogía de Educación Básica en período de prácticas profesionales, de tres universidades acreditadas en carreras ubicadas en tres regiones de Chile. Se filmaron dos clases consecutivas que fueron analizadas con categorías del análisis de la conversación. Se observó que las estructuras de conversación descritas están asociadas a tipos de intervención que hace el estudiante de Pedagogía en la clase. Adicionalmente se observó que la organización de la clase es similar en varios aspectos a las clases que realizan los profesores en servicio y con más trayectoria en el campo profesional.

Palabras clave: Formación de docentes, relación profesor-alumno, práctica pedagógica.

\section{Abstract}

Professional practice in initial teacher training provides situated knowledge of teaching. This paper analyzes classes taught by student teachers; six students were observed as they performed their internship for the basic-level education pedagogy degree from three accredited universities in three regions of Chile. Two consecutive classes were filmed and analyzed using conversation analysis categories. It was found that the conversation structures described are associated with types of intervention by pedagogy students in the classroom. It was also noted that the organization of classes was similar in many respects to classes by in-service teachers with more professional experience.

Keywords: Teacher education, student teacher relationship, teaching practice.

\footnotetext{
${ }^{1}$ Estudio auspiciado por el Fondo Nacional de Desarrollo Científico y Tecnológico (FONDECYT) del gobierno de Chile, No. 1150931
} 


\section{Introducción}

La formación inicial de profesores en América Latina se encuentra en un profundo proceso de cambios, los cuales se producen en la definición del perfil de egreso, en el currículo, la pedagogía y en la organización institucional de las universidades e institutos de formación para favorecer el desarrollo profesional (Bruns y Luque, 2014; De Gregorio y Bruns, 2016; Vaillant, 2013). Con estas transformaciones se espera que los nuevos profesores tengan las competencias que se requieren para enfrentar los desafíos de calidad y aprendizaje que demanda la sociedad actual.

La formación de profesores siempre ha tenido actividades prácticas que, tradicionalmente, se ubicaban al final de la formación teórica o disciplinar. Hoy día, la tendencia es otorgar una centralidad mayor a la práctica iniciándola en los primeros años de la formación. Se define como "práctica progresiva", ubicando distintos tipos de actividades en el currículo que implican observaciones, entrevistas y trabajo real en las escuelas desde los primeros años de estudio (Ferrada et al., 2013; Hepp, 2015; Pozo et al., 2016).

Las prácticas profesionales activan un saber situado de la enseñanza. Las universidades organizan un dispositivo pedagógico que implica, entre otros, selección de supervisores; comunicación y coordinaciones con los centros de práctica, la selección y formación de los profesores guías o colaboradores, el diseño de las actividades prácticas y la reflexión pedagógica de las mismas. Las relaciones entre universidades y establecimientos educativos no son fáciles y muchas veces ponen de manifiesto las distancias que existen entre la formación inicial y la realidad de la labor docente profesional en el aula. Incluso suele ocurrir que los futuros profesores tienden a movilizar saberes de sus propias experiencias escolares para enfrentar situaciones en el aula para las cuales no han sido preparados (Gariglio y Burnier, 2014; Martinic y Villalta, 2015).

Las clases realizadas por los futuros profesores suelen ser observadas por supervisores y docentes universitarios, quienes evalúan el desempeño en función del currículo formativo y pautas previamente definidas (Andreucci-Annunziata, 2013; Solís et al., 2011). Sin embargo, suele haber una brecha entre los cursos teóricos ofrecidos en los programas de formación inicial y el ejercicio profesional (Martinic et al., 2014; Saiz et al., 2014; Solís et al., 2011); Por lo general, en la supervisión se observan sólo alguna de las clases realizadas por los estudiantes de pedagogía y las pautas de evaluación suelen estar centradas en el manejo conceptual y didáctico esperado y definido por las instituciones formativas más que en la experiencia real de la práctica que han tenidos los futuros profesores.

Por ejemplo, la observación de las interacciones con los alumnos, la comunicación que se establece en el aula, el manejo de tiempo y la resolución de conflictos en el aula, entre otros aspectos de la experiencia práctica, no están suficientemente integradas en la observación y evaluación que realizan los supervisores de las actividades prácticas de la formación inicial (Sánchez y Jara, 2016; Steins et al., 2015).

Investigaciones como las de Andreucci-Annunziata (2013), Stough et al. (2015) y Martinic y Villalta (2016) han demostrado la importancia de la gestión del tiempo y la comunicación efectiva para el aprendizaje del oficio docente y, por ello, recomiendan su estudio y observación en la formación inicial de profesores.

Por otra parte, y desde una perspectiva sociocultural, es importante poner atención en los estilos comunicativos de los profesores, en su capacidad de generar diálogos y participación de los estudiantes más allá de la mera repetición o confirmación de lo enunciado por los docentes (Martinic y Villalta, 2016; Pasmanik y Cerón, 2005; Preiss et al., 2016). Ello es importante porque el diálogo y la comunicación profesor-alumno son dimensiones claves del clima de aula y factores determinantes para los aprendizajes. El estudiante de pedagogía necesita desarrollar las competencias comunicativas que le permitan el manejo de las conductas disruptivas y de indisciplina en el aula para el desarrollo de las estructuras comunicativas de la clase (Hamilton, 2015; Harding y Hbaci, 2015; Macías y Sánchez, 2015).

La estructura básica del diálogo en el aula supone intervenciones de Inicio-Respuesta-Feedback o retroalimentación IRF, descrita en la literatura inglesa por Sinclair y Coulthard (1975) y definida en la investigación francófona como "clase dialogada" por Veyrunes y San Martín (2016). La unidad mínima del 
diálogo se define como intercambio, esto es, intervenciones de interlocutores diferentes mutuamente orientados que contribuyen a desarrollar un tema (Hennessy et al., 2016).

La estructura IRF, ocupa gran parte del tiempo de la clase (Veyrunes y San Martín, 2016; Villalta et al., 2013). Diversos autores han descrito variantes de la estructura IRF identificando, por ejemplo -según la cantidad de intervenciones- intercambios simples o complejos, o bien -según la demanda cognitiva del diálogointercambios co-formados, expositivos e instruccionales, entre otros, (Davin, 2013; Nathan y Kim, 2009; Veyrunes y San Martín, 2016).

Teniendo en cuenta el número de intervenciones, los intercambios pueden ser simples o complejos. Los intercambios simples son diálogos compuestos por dos o tres intervenciones a través de los cuales los interlocutores producen una satisfacción interaccional. Esto es, llegan a un acuerdo que satisface los requerimientos iniciales que dan origen al intercambio. Por ejemplo, el profesor pregunta, el estudiante responde el contenido esperado y el profesor evalúa positivamente la respuesta.

Los intercambios complejos tienen una mayor cantidad de intervenciones, ya que para alcanzar la satisfacción interaccional de los interlocutores existe una negociación de sentidos más compleja. Por ejemplo, cuando los profesores evalúan en forma negativa la respuesta a una pregunta y construyen la respuesta correcta con intervenciones sucesivas del mismo alumno u otros diferentes que cooperan en la solución (Martinic et al., 2013).

En cuanto a la demanda cognitiva, los intercambios pueden ser considerados como de alta y baja complejidad cognitiva (Martinic et al., 2013; Villalta et al., 2013). Los de baja complejidad, por lo general, son intercambios donde la participación del alumno es receptiva o responsiva a las demandas de la intervención del profesor. Es el caso del intercambio expositivo o instruccional, donde la intervención de los alumnos validan la intervención del profesor; intercambios para regular el orden de los turnos de participación e intercambios co-formados, donde el profesor verifica en los alumnos el dominio de un saber previamente transmitido en la clase.

Los intercambios de alta complejidad cognitiva, en cambio, refieren a aquellos en los cuales los estudiantes toman mayor iniciativa en la co-construcción de significados (Wells y Mejía-Arauz, 2005) que constituyen conocimiento escolar, tales como: los intercambios explicativos, donde el estudiante inicia el intercambio solicitando al profesor mayor información sobre lo trabajado en la clase; intercambios cooperativos, donde los estudiantes participan junto con el profesor co-construyendo los contenidos de la clase, e intercambios colaborativos, donde los estudiantes intervienen movilizando su experiencia para dialogar de su propio aprendizaje en la clase. En todos los casos la descripción de alta o baja complejidad cognitiva refiere a las características y posibilidades de la interacción y los intercambios, como expresión de los procesos cognitivos de los alumnos.

En este artículo se analiza y compara el uso del tiempo de los intercambios profesor-alumnos en las clases realizadas por futuros profesores en dos contextos socioculturales diferentes, y que corresponden a la actividad curricular de práctica profesional en el último año de formación. Los resultados permiten profundizar en la importancia que tiene el contexto sociocultural en la configuración de las estructuras comunicacionales en el aula y en los patrones comunes de comunicación, específicamente en la práctica profesional.

\section{Método}

Estudio descriptivo de los usos del tiempo en los intercambios profesor-alumnos en el aula a través de la observación no participante con registro fílmico de clases reales. Los escenarios de estudio son escuelas de Educación Básica, que son centro de práctica de tres universidades de tres regiones de Chile con realidades socioculturales diferentes: la Región Metropolitana pertenece a una realidad netamente urbana, en tanto las regiones de la Araucanía (Región 2) y Coquimbo (Región 1) tienen menor densidad poblacional y mayor componente rural y étnico en comparación con la escuela de la Región Metropolitana. Se observó a dos estudiantes de Pedagogía en Educación Básica por región, trabajando en total con seis estudiantes de 
Pedagogía de 5o. año, como se registra en la tabla l.

Tabla I. Escenario y sujetos de estudio por género

\begin{tabular}{lccc}
\hline \multirow{2}{*}{ Casos } & \multicolumn{2}{c}{ Género } & \multirow{2}{*}{ Total } \\
\cline { 2 - 3 } & Mujer & Hombre & \\
\hline Región 2 (Araucanía) & 1 & 1 & 2 \\
Región Metropolitana & 1 & 1 & 2 \\
Región 1 (Coquimbo) & 2 & - & 2 \\
Total & 4 & 2 & 6 \\
\hline
\end{tabular}

\subsection{Procedimiento de Análisis}

Los videos se codificaron según categorías de distribución del tiempo de la clase a diferentes niveles de la interacción profesor-alumno. Dichas categorías están sustentadas y descritas en el de estudio de Martinic y Villalta (2015, p. 49), del cual se toman los niveles de Fases, Dirección del habla, Intervenciones e Intercambios, que a continuación se definen brevemente. Las Fases describen las grandes etapas o segmentos de una clase y que van desde el pre-inicio al cierre o término de la misma; la Dirección del habla del profesor, según sea al grupo, a un alumno, todo el curso; la Intervención del profesor, como partícipe en el diálogo pedagógico con los alumnos. Las Intervenciones describen desde el inicio, aquel que abre la conversación, el desarrollo del diálogo, hasta el cierre, aquella intervención que da por terminada la unidad dialogal al alcanzar la satisfacción interaccional. En este artículo se analizan los tipos de intervención de los profesores en el inicio, desarrollo y cierre del intercambio.

Los intercambios son la unidad dialogal mínima de la conversación, en el contexto escolar han sido descritas como de tipo expositivo, co-formado, regulativo, explicativo, cooperativo y colaborativo (Villalta et al. 2013, p. 91). La concordancia inter-observador realizada entre dos codificadores independientes se analizó con índice Kappa, el cual va de .723 a .736, lo que indica Buena Concordancia (las categorías se describen en el Anexo).

Dado el carácter nominal y ordinal de los datos categorizados, así como el número de participantes, el análisis se realizó con estadísticos no paramétricos para comparar la distribución de las categorías observadas según la región, y estudiante de pedagogía. Se aplicó la Prueba de Mann-Whitney para comparar la distribución entre dos grupos definidos, por ejemplo, la región gegráfica o contexto donde se realiza la práctica profesional; y la prueba Kruskal-Wallis para comparar más de dos grupos, por ejemplo, comparar las categorías de estudio del aula entre los seis estudiantes

Asimismo, se analizó la asociación entre las categorías observadas en el aula (ver tabla II), entre éstas y la región geográfica de la práctica, y estudiante de pedagogía en práctica; utilizando el estadístico Chicuadrado, y analizando la fuerza de dicha asociación con la $V$ de Cramer, coeficiente que va de 0 = nula fuerza de la asociación a 1 = fuerte asociación; en Ciencias Sociales un valor V de Cramer por sobre 30 puede ser considerado como de asociación de fuerza moderada; y fuerte asociación por sobre .50; se considera débil bajo 29 y nula de .10 a menos.

\section{Resultados}

A continuación se describen los usos del tiempo en las Fases de la clase, Dirección del habla del profesor, Intercambios e Intervenciones observadas en los practicantes agrupados según las tres universidades formadoras. Se comparan los resultados entre ellas y posteriormente se analiza la relación entre los practicantes según universidad y tipos de intercambios registrados. Se compara la distribución de las categorías entre las regiones donde se realiza la práctica y, finalmente, se analiza la relación de los intercambios según el tipo de administración de la universidad. 
Tabla II. Frecuencia porcentual de las categorías Fases por región

\begin{tabular}{|c|c|c|c|c|}
\hline \multicolumn{5}{|c|}{ Universidad (\%) } \\
\hline Fases de la clase & Regional 1 & Metropolitana & Regional 2 & Total \\
\hline Preinicio & 0.2 & 10.6 & 1.2 & 2.2 \\
\hline Inicio & 2.3 & 10.2 & 6.8 & 5.3 \\
\hline Desarrollo & 91.3 & 78.5 & 88.9 & 88.4 \\
\hline Cierre pedagógico & 4.3 & & 3.1 & 3.2 \\
\hline Cierre & 1.8 & 0.7 & & 0.9 \\
\hline Total \% & 100 & 100 & 100 & 100 \\
\hline N total (segundos) & 4,338 & 1,475 & 4,048 & 9,861 \\
\hline \multicolumn{5}{|l|}{ Dirección del habla del profesor } \\
\hline A la clase & 42.9 & 54.8 & 30.9 & 39.7 \\
\hline Al grupo & 0.2 & 1.2 & 4.4 & 2.1 \\
\hline A un alumno & 19.4 & 12.7 & 28.5 & 22.2 \\
\hline Sin intervención & 37.4 & 31.3 & 36.2 & 36.0 \\
\hline Total & 100 & 100 & 100 & 100 \\
\hline N total (segundos) & 4,338 & 1,475 & 4,088 & 9,901 \\
\hline \multicolumn{5}{|l|}{ Intercambios } \\
\hline Expositivo & 24.0 & 19.1 & 27.6 & 24.8 \\
\hline Co-formado & 36.9 & 23.5 & 29.0 & 31.9 \\
\hline Regulativo & 3.1 & 29.6 & 36.4 & 20.1 \\
\hline Explicativo & 7.2 & 5.6 & 5.1 & 6.2 \\
\hline Cooperativo & 25.9 & 21.5 & 1.2 & 15.3 \\
\hline Colaborativo & 2.9 & 0.7 & 0.6 & 1.7 \\
\hline Total \% & 100 & 100 & 100 & 100 \\
\hline N total (segundos) & 4,168 & 1,204 & 3,663 & 9,035 \\
\hline \multicolumn{5}{|l|}{ Intervención del profesor } \\
\hline Exposición & 36.6 & 21.4 & 33.7 & 33.2 \\
\hline Preguntas & 29.7 & 22.6 & 24.7 & 26.5 \\
\hline Regulación disciplinar & 0.9 & 12.4 & 10.0 & 6.4 \\
\hline Regulación de procedimiento & 7.5 & 21.8 & 21.0 & 15.3 \\
\hline Responder al alumno & 25.3 & 21.8 & 10.6 & 18.6 \\
\hline Total \% & 100 & 100 & 100 & 100 \\
\hline N total (segundos) & 2,640 & 886 & 2,594 & 6,120 \\
\hline
\end{tabular}

Como se muestra en la tabla II, en las clases de la región Metropolitana los practicantes ocuparon porcentualmente más tiempo en las Fases de Preinicio e Inicio de la clase, sus intervenciones se dirigieron a la clase como grupo (más del 50\% del tiempo) y realizaron más intervenciones de Regulación disciplinar, en comparación con las prácticas de las universidades regionales. Por su parte, los practicantes de Universidades Regionales dedicaron más tiempo para implementar la fase de Cierre pedagógico, la dirección del habla fue hacia un alumno específico, y en más del $30 \%$ del tiempo de la clase no se dirigieron específicamente a alumno alguno, se dieron más intercambios Expositivos y Co-formados, y más del 50\% de la intervención de los profesores fue de tipo Preguntas y Exposición, en comparación con los profesores de la universidad de la región Metropolitana. 
Tabla III. Prueba de Kruskal-Wallis de las categorías para comparar entre universidades

\begin{tabular}{l|c|c|c|c}
\hline Estadísticos de contraste $(\mathbf{a} \mathbf{y}$ b) & $\begin{array}{c}\text { Dirección del } \\
\text { habla } \\
\text { del profesor }\end{array}$ & Intercambios & $\begin{array}{c}\text { Intervención } \\
\text { del profesor }\end{array}$ \\
\hline chase & 120.792 & 187.495 & 78.803 \\
gl & 574.159 & 2 & 2 & 2 \\
Sig. asintót. & 2 & .001 & .001 & .001 \\
\hline
\end{tabular}

Nota: a) Prueba de Kruskal-Wallis, y b)Variable de agrupación: Universidad

Considerando que las distribuciones de tiempo de las categorías de observación son significativamente diferentes entre los practicantes agrupados por universidad formadora, interesa conocer si dichas diferencias tienen que ver con asociación específica entre las categorías de observación de aula y atributos de los casos. El análisis con estadístico de asociación Chi cuadrado entre todas las categorías observadas (Fases, Dirección del habla del profesor e Intervenciones) y Universidad, indican asociación significativa; pero la fuerza de esta asociación es Débil (V de Cramer < .30). En todas, excepto en la categoría Intercambio.

Tabla IV. Intercambios por región de la Universidad

\begin{tabular}{l|c|c|c|c|c}
\hline \multirow{2}{*}{ Intercambios } & \multicolumn{4}{c}{ Universidad (\%) } \\
\cline { 3 - 5 } Expositivo & $\%$ & 24.0 & 19.1 & 27.6 & 24.8 \\
& Residuos corregidos & -1.6 & -4.9 & 5.1 & \\
Co-formado & $\%$ & 36.9 & 23.5 & 29.0 & 31.9 \\
& Residuos corregidos & 9.4 & -6.7 & -4.9 & \\
Regulativo & $\%$ & 3.1 & 29.6 & 36.4 & 20.1 \\
& Residuos corregidos & -37.4 & 8.8 & 31.9 & \\
Explicativo & $\%$ & 7.2 & 5.6 & 5.1 & 6.2 \\
& Residuos corregidos & 3.9 & -0.9 & -3.3 & \\
Cooperativo & \% & 25.9 & 21.5 & 1.2 & 15.3 \\
& Residuos corregidos & 25.8 & 6.4 & -30.7 & \\
\multirow{2}{*}{ Colaborativo } & \% & 2.9 & 0.7 & 0.6 & 1.7 \\
& Residuos corregidos & 8.3 & -2.7 & -6.6 & \\
\hline \multirow{2}{*}{ Total } & \% & 100 & 100 & 100 & 100 \\
& Recuento & 4,168 & 1,204 & 3,663 & 9,035 \\
\hline
\end{tabular}

Hay asociación significativa [Chi Cuadrado $(10, N=9,035)=2126,981 ; p<.001$ ], y de fuerza moderada ( $V$ de Cramer $=.343, p<.001$ ) entre el tipo de intercambios y la Universidad (tabla IV); es decir, las estructuras de intercambio observadas son diferentes entre los practicantes agrupados por región de la universidad (tabla III), y además están relacionadas a los micro-contextos de aula donde los practicantes participan (tabla IV).

En el análisis de las casillas a través de los Residuos tipificados (valor $z= \pm 1.96$ calculado entre los frecuencias observadas y esperadas en cada casilla; así, cuanto mayor es el valor de z mayor es la fuerza positiva/negativa de la asociación en la casilla) tipos de Intercambio y universidad, se encuentra que los practicantes de la universidad Regional 1 tienden a generar Intercambios de tipo Cooperativo y Coformados; los practicantes de la región Metropolitana, en cambio, se caracterizan por generar Intercambios Regulativos y Cooperativos; y los practicantes de la Región 2 se caracterizan por generar Intercambios Regulativos y Expositivos. A continuación se presentan ejemplos de los cuatro intercambios que caracterizan a los contextos regionales de las aulas observadas: 


\section{Ejemplo 1.}

Intercambios: EXPOSITIVO: Clase de Matemáticas.

Región 2 (Araucanía)

00:51:39-00:52:06

Profesor:<Al finalizar la clase de matemáticas, donde jugaron en parejas con un dado, para llenar una matriz de probabilidades de números así como funciona este juego, funcionan muchas cosas en la vida, como puede ser cuando van a los casinos o cuando juegan un loto o un kino, este es el método como trabajan ellos, hay una pequeña probabilidad de que salga un evento más que el otro, en este caso, el evento B es menos probable de que salga acá, ellos trabajan así chicos.

Alumnos: <En silencio, los siete alumnos escuchan al profesor>

\section{Ejemplo 2.}

Intercambio REGULATIVO: Clase de matemáticas.

Región 2 (Araucanía)

00:03:21 - 00:03:23

Profesora:<Dirigiéndose a una alumna> Andrea, siéntate hija.

Andrea: <estaba apoyada en su mesa, se sienta>

profesora: Ya.

\section{Ejemplo 3.}

Intercambio CO-FORMADO: Clase de Geografía.

Región 1 (Coquimbo)

00:11:56 - 00:12:24

Profesora: <Revisa dominio de contenidos por los alumnos, mientras estos trabajan preparando una maqueta sobre geografía de Chile, que trabajan en la clases. Yanara, cuéntenos que es lo que va a hacer con su maqueta para hoy día.

Yanara: (Algo nerviosa) ... del relieve.

Profesora: Ya... del relieve, ¿cuáles son las cuatro formas principales de relieve?, ¿se acuerda?

Yanara: ¿La cordillera de los Andes?

Profesora: Yaaa.

Yanara: La depresión intermedia.

Profesora: La depresión intermedia.

Yanara: La cordillera de la costa.

Profesora: Yaa.

Yanara: Y las planicies litorales.

Profesora:Ya!

\section{Ejemplo 4.}

Intercambio COOPERATIVO: Clase de Matemáticas.

Región 1 (Coquimbo)

00:02:16 - 00:02:40

Profesora: <Al inicio de la clase>. ¿Cuál es la gran diferencia que hay entre una ecuación y una expresión algebraica?

Alumna: Ehh, las ecuaciones son las cortas que las expresiones algebraicas.

Profesora: Ya, las ecuaciones son más cortas, ¿alguien tiene otra diferencia?

Alumno: La ecuación son igual a los resultados.

Profesora: La ecuación es igual a los resultados, muy bien. 


\section{Ejemplo 5.}

Intercambio REGULATIVO: Clase Lenguaje

Región Metropolitana

00:25:54 - 00:26:05

Profesor: Sigo escuchando voces a quienes no he dado la palabra /

Alumno: ¿Puedo decir algo?

Profesor: Un momento, necesito silencio.

\section{Ejemplo 6.}

Intercambio COOPERATIVO. Clase Lenguaje

Región Metropolitana

00:33:31 - 00:33:44

Profesor: Si, por ejemplo, yo digo que la poesía está escrita en estrofas, en versos, ¿cómo está escrita la obra dramática?

Alumnos: <Varios levantan la mano>. ¡Por diálogos!

Profesor: ¡Con diálogos! Súper.

Los intercambios de los ejemplos 1 al 6 representan las estructuras mayoritarias en las clases observadas en cada región. Las estructuras Cooperativas (ejemplos 4 y 6) de la Región 1 (Coquimbo) y Metropolitana son posibles en aulas de alto rendimiento escolar, ya que ponen de manifiesto un uso de lenguaje especializado y de dominio de contenidos ya incorporados en las intervenciones de los alumnos. Las estructuras regulativas se orientan al logro del orden (ejemplos 2 y 5) en el primer caso (ejemplo 2) se trata de lograr el orden de uso del espacio en un aula rural (región de la Araucanía) y en el segundo caso (ejemplo 5) se trata de lograr el orden de los turnos de habla en un aula con aproximadamente 40 alumnos en la sala (región Metropolitana).

En la tabla $V$ se agrupan las Universidades Formadoras en dos grupos según administración: 1) Estatal (Regional 1 + Metropolitana), y 2) Privada ( Regional 2).

Tabla V . Prueba U de Mann-Whitney de las categorías para comparar dos tipos de administración de universidades

\begin{tabular}{|c|c|c|c|c|c|c|c|}
\hline & & & \multicolumn{2}{|c|}{ Rangos } & \multicolumn{3}{|c|}{ Estadísticos de contraste ${ }^{*}$} \\
\hline $\begin{array}{l}\text { Categorías } \\
\text { de observación }\end{array}$ & Administración & $\mathrm{N}$ & $\begin{array}{c}\text { Rango } \\
\text { promedio }\end{array}$ & $\begin{array}{c}\text { Suma de } \\
\text { rangos }\end{array}$ & $\begin{array}{l}\text { U de Mann- } \\
\text { Whitney }\end{array}$ & $\mathrm{Z}$ & $\begin{array}{c}\text { Sig. } \\
\text { asintót. } \\
\text { (bilateral) }\end{array}$ \\
\hline \multirow{3}{*}{$\begin{array}{l}\text { Fases } \\
\text { de la clase }\end{array}$} & Estatal & 5,813 & $4,975.463$ & 28922366 & \multirow{3}{*}{11507049} & \multirow{3}{*}{$-3,346$} & \multirow{3}{*}{.001} \\
\hline & Privada & 4,048 & $4,867.150$ & 19702225 & & & \\
\hline & Total & 9,861 & & & & & \\
\hline \multirow{3}{*}{$\begin{array}{l}\text { Dirección } \\
\text { del habla } \\
\text { del profesor }\end{array}$} & Estatal & 5,813 & $4,763.146$ & 27688167 & \multirow{3}{*}{10789776} & \multirow{3}{*}{$-8,314$} & \multirow{3}{*}{.001 } \\
\hline & Privada & 4,088 & $5,218.122$ & 21331684 & & & \\
\hline & Total & 9,901 & & & & & \\
\hline \multirow{3}{*}{ Intercambios } & Estatal & 5,372 & $4,801.413$ & 25793193 & \multirow{3}{*}{8316321} & \multirow{3}{*}{$-12,899$} & \multirow{3}{*}{.001} \\
\hline & Privada & 3,663 & $4,102.358$ & 15026937 & & & \\
\hline & Total & 9,035 & & & & & \\
\hline \multirow{3}{*}{$\begin{array}{l}\text { Intervención } \\
\text { del Profesor }\end{array}$} & Estatal & 3,526 & $3,129.888$ & 11035985 & \multirow{3}{*}{4328560} & \multirow{3}{*}{$-3,705$} & \multirow{3}{*}{.001} \\
\hline & Privada & 2,594 & $2,966.1816$ & 7694275 & & & \\
\hline & Total & 6,120 & & & & & \\
\hline
\end{tabular}

Nota: *Variable de agrupación: Administración

Se compara la distribución de las categorías de observación entre las universidades agrupadas según Administración (tabla V). El estadístico de contraste U de Mann-Whitney indica que es significativamente diferente la distribución del tiempo entre los practicantes agrupados por universidad. 
Se reitera en el análisis de asociación con estadístico Chi cuadrado entre todas las categorías observadas y la nueva categoría generada: Administración de la Universidad. Se encuentra asociación significativa, pero en la gran mayoría de los intercambios la fuerza de esta asociación es Débil (V de Cramer <.30). En todas, excepto en la categoría Intercambio.

Tabla VI. Intercambios por tipo de administración de la Universidad

\begin{tabular}{|c|c|c|c|c|}
\hline \multirow{2}{*}{\multicolumn{2}{|c|}{ Intercambios }} & \multicolumn{2}{|c|}{ Administración (\%) } & \multirow{2}{*}{ Total } \\
\hline & & Estatal & Privada & \\
\hline \multirow{2}{*}{ Expositivo } & $\%$ & 22.9 & 27.6 & 24.8 \\
\hline & Residuos corregidos & -5.1 & 5.1 & \\
\hline \multirow{2}{*}{ Co-formado } & $\%$ & 33.9 & 29.0 & 31.9 \\
\hline & Residuos corregidos & 4.9 & -4.9 & \\
\hline \multirow{2}{*}{ Regulativo } & $\%$ & 9.0 & 36.4 & 20.1 \\
\hline & Residuos corregidos & -31.9 & 31.9 & \\
\hline \multirow{2}{*}{ Explicativo } & $\%$ & 6.9 & 5.1 & 6.2 \\
\hline & Residuos corregidos & 3.3 & -3.3 & \\
\hline \multirow{2}{*}{ Cooperativo } & $\%$ & 24.9 & 1.2 & 15.3 \\
\hline & Residuos corregidos & 30.7 & -30.7 & \\
\hline \multirow{2}{*}{ Colaborativo } & $\%$ & 2.4 & 0.6 & 1.7 \\
\hline & Residuos corregidos & 6.6 & -6.6 & \\
\hline \multirow{2}{*}{ Total } & $\%$ & 100 & 100 & 100 \\
\hline & $\mathrm{N}$ & 5,372 & 3,663 & 9,035 \\
\hline
\end{tabular}

Se observa asociación significativa entre los tipos de Intercambio y la administración de la universidad [Chi Cuadrado $(5, N=9,035)=1,698.898 ; p<.001]$, de fuerza moderada (V de Cramer $=.434)$ (tabla VI). Es decir, las estructuras de intercambio observadas son diferentes entre los practicantes agrupados por universidad y región (ver tabla III), no obstante éstas se encuentran relacionadas al tipo de administración de la universidad (tabla VI).

El análisis de los Residuos tipificados de las casillas pone en evidencia que en la universidad Privada se dieron con mayor fuerza intercambios Expositivos y Regulativos -juntas ocupan alrededor del 64\% del tiempo de intercambios observados-; esto significa que la participación de los alumnos en estas aulas fue de carácter mayoritariamente receptivo de los contenidos y actividades de enseñanza desarrollados por los practicantes. En tanto en la Universidad Estatal se dieron intercambios Co-formados, Explicativos, Cooperativos y Colaborativos -que juntos representan el $68.1 \%$ del tiempo de los intercambios observados-, lo cual significa que la participación de los alumnos se diversificó entre responder a las demandas de los practicantes y tomar la iniciativa en unos casos, o movilizar la propia experiencia para participar de la clase.

En los seis estudiantes para profesor en período de práctica profesional se observa una asociación significativa (con uso de estadístico Chi cuadrado) y de Fuerza Moderada (V de Cramer > .30 y <.50) entre tipos de Intervenciones tanto de inicio, desarrollo y cierre de la conversación con los tipos de Intercambios. 
Tabla VII. Intercambios por Intervenciones en el total de casos

\begin{tabular}{|c|c|c|c|c|c|c|c|}
\hline \multirow{2}{*}{ Intercambios } & & \multicolumn{5}{|c|}{ Intervención del profesor (\%) } & \multirow[b]{2}{*}{ Total } \\
\hline & & Exposición & Preguntas & $\begin{array}{c}\text { Regulación } \\
\text { disciplinar }\end{array}$ & $\begin{array}{l}\text { Regulación de } \\
\text { procedimiento }\end{array}$ & $\begin{array}{l}\text { Responder } \\
\text { al alumno }\end{array}$ & \\
\hline \multirow{2}{*}{ Expositivo } & $\%$ & 65.8 & 3.2 & 2.8 & 15.4 & 33.3 & 31.4 \\
\hline & R. corregidos & 40.8 & -28.5 & -12.6 & -11.5 & 1.6 & \\
\hline \multirow{2}{*}{ Co-formado } & $\%$ & 17.8 & 65.4 & 7.7 & 6.7 & 29.9 & 30.4 \\
\hline & R. corregidos & -15.0 & 35.8 & -10.1 & -17.1 & -0.3 & \\
\hline \multirow{2}{*}{ Regulativo } & $\%$ & 2.7 & 11.4 & 87.4 & 65.1 & 7.7 & 20.9 \\
\hline & R. corregidos & -24.7 & -11.0 & 33.4 & 36.0 & -12.1 & \\
\hline \multirow{2}{*}{ Explicativo } & $\%$ & 6.5 & 1.5 & 0.3 & 3.9 & 14.1 & 5.8 \\
\hline & R. corregidos & 1.7 & -8.6 & -4.8 & -2.7 & 13.3 & \\
\hline \multirow{2}{*}{ Cooperativo } & $\%$ & 7.1 & 15.9 & 1.8 & 8.9 & 13.2 & 10.5 \\
\hline & R. corregidos & -6.1 & 8.2 & -5.8 & -1.7 & 3.3 & \\
\hline \multirow{2}{*}{ Colaborativo } & $\%$ & 0.1 & 2.7 & 0.0 & 0.1 & 1.8 & 1.1 \\
\hline & R. corregidos & -5.0 & 7.0 & -2.2 & -3.2 & 2.4 & \\
\hline \multirow{2}{*}{ Total } & $\%$ & 100 & 100 & 100 & 100 & 100 & 100 \\
\hline & $\mathrm{N}$ & 2,022 & 1,620 & 390 & 931 & 1,122 & 6,085 \\
\hline
\end{tabular}

La asociación significativa entre tipos de Intercambios con tipos de Intervención se observa también en el conjunto general de los datos [Chi Cuadrado $(20, N=6,085)=4,917.736 ; p<.001$ ], con fuerza de asociación Moderada (Chi Cuadrado significativo, V de Cramer = .449) (ver Tabla VII).

El análisis de los Residuos tipificados de las casillas, complementada con el análisis de distribución porcentual, pone en evidencia que la Intervención del profesor "Responder al alumno" está asociada con tres tipos de intercambios considerados de alta demanda cognitiva: Explicativo, Cooperativo y Colaborativo, y la Intervención "Preguntas" con los dos últimos intercambios más el intercambio Coformado. Por su parte, la intervención "Exposición" está fuertemente relacionada con el intercambio Expositivo. En efecto, Exponer, Preguntar y Responder a los alumnos representan el $78.3 \%$ del tiempo de las intervenciones del profesor; las intervenciones Preguntar y Responder alrededor del 45\% del tiempo de las intervenciones observadas y están asociadas a tipos de intercambio que requieren mayor participación e iniciativa de los alumnos (Co-formados, Explicativos, Cooperativos y Colaborativos).

\section{Discusión y conclusiones}

Los resultados presentados sobre distribución del tiempo en las fases de la clase indican que aproximadamente el $80 \%$ del tiempo corresponde a la fase de Desarrollo donde ocurren, principalmente, intervenciones asociadas a contenidos instruccionales. Este dato se aproxima a las proporciones encontradas en estudios realizados por el Banco Mundial y que son considerados como un referente para el uso del tiempo en la clase (Bruns y Luque, 2014; De Gregorio y Bruns, 2016).

El uso del tiempo de la clase en todas las categorías descritas (Fases, Intervenciones e Intercambios), aunque diferente entre los estudiantes para profesor observadas, sigue la tendencia de gestión de tiempo de los profesores en servicio profesional: las fases se distribuyen de modo similar a las observadas en escuelas de Educación Básica Urbanas con Jornada Escolar Completa (JEC) que han tenido alto logro escolar en la prueba nacional SIMCE (Sistema de Evaluación de la Calidad de la Educación) (Martinic y Villalta, 2015; 2016), donde también la intervención mayoritaria del profesor es de tipo Expositivo (Martinic y Villalta, 2015; 2016).

En tal sentido, es posible considerar que las configuraciones de uso del tiempo de los estudiantes de Pedagogía en Educación Básica son expresión de la "fuerza cultural de la clase dialogada" (Veyrunes y San Martín, 2016) respecto a la hegemonía de un patrón comunicacional de transmisión de saberes escolares 
que se valida constantemente a través de preguntas; y de los efectos de la política pública educativa con respecto a los estándares de formación inicial de los profesores en período de práctica profesional. No obstante, como los datos también lo indican, las prácticas de aula son construcciones contextualizadas; en consecuencia, la planificación de estándares de formación inicial debe considerar el carácter situacional y complejo de la realidad educativa (Aguerrondo, 2014).

Considerando que las estructuras de intercambio y de uso del tiempo que caracterizan la práctica de los estudiantes para profesor son similares en aspectos generales a la gestión del tiempo de la clase de los profesores en servicio, se pone en evidencia, por una parte, la necesidad de tener la clase dialogada como un objeto de la formación inicial docente (Steins, et al., 2015; Veyrunes y San Martín, 2016), y por otra parte, analizar las condiciones teóricas y socio-temporo-espaciales para promover la práctica docente en el aula que gestione la participación y aprendizaje en contextos de diversidad cultural (Christofferson y Sullivan, 2015; Glock, 2016).

La actividad de los estudiantes para profesor posee patrones dialogales en la sala de clase que son diferentes al comparar por ubicación urbano/rural de la escuela. En efecto, se observa asociación moderada entre los intercambios comunicativos en el aula y el contexto, lo cual permite afirmar que se trata de acciones contextualizadas que, no obstante, tienden a realizar una distribución temporal de las actividades de la clase de modo similar a como se trabaja en escuelas de alto rendimiento de aprendizaje escolar.

En conclusión, en el período de práctica profesional docente, la distribución de uso del tiempo de la clase es similar a nivel de las fases de la clase y los tipos de intervenciones del profesor, pero con tendencias diferenciadoras en el micro nivel de los intercambios comunicativos, asociados al tipo de contexto sociocultural de realización de la práctica.

Futuros estudios deben indagar la relación entre los modelos de supervisión y el tipo de práctica de aula que configuran los estudiantes para profesor, considerando el papel del contenido de asignatura como un elemento que abre y cierra posibilidades de interacciones comunicativas. Asimismo, es necesario analizar con mayor precisión las similitudes y diferencias entre la actividad del docente en servicio y las del estudiante para docente en el aula, de tal forma que se determine cuáles son las habilidades comunicativas que se promueven para construir y sostener los patrones dialogales tradicionales, y cuáles dejan espacio para la innovación de respuestas a la realidad de diversidad cultural en las aulas.

\section{Referencias}

Aguerrondo, I. (2014). Planejamento educativo e complexidade: gestão das reformas educacionais [Planificación educativa y complejidad: gestión de las reformas educativas]. Cadernos de Pesquisa, 44(153), 548-578. http://publicacoes.fcc.org.br/ojs/index.php/cp/article/view/2910

Andreucci-Annunziata, P. (2013). La supervisión de prácticas docentes: una deuda pendiente de la formación inicial de profesores. Estudios Pedagógicos, 39(1), 7-26.

http://revistas.uach.cl/index.php/estped/article/view/2596

Bruns, B. y Luque, J. (2014). Profesores excelentes: cómo mejorar el aprendizaje en América Latina y el Caribe. Banco Mundial. https://dds.cepal.org/redesoc/publication?id=3806

Christofferson, M. y Sullivan, A. L. (2015). Preservice teachers' classroom management training: a survey of self-reported training experiences, content coverage, and preparedness. Psychology in the Schools, 52(3), 248-264. https://doi.org/10.1002/pits.21819

Davin, K. J. (2013). Integration of dynamic assessment and instructional conversations to promote development and improve assessment in the language classroom. Language Teaching Research, 17(3), 303322. https://doi.org/10.1177/1362168813482934 
De Gregorio, S. y Bruns, B. (2016). Práctica docente en el aula: una mirada al interior de las salas de clases en América Latina y el Caribe. En J. Manzi y M. R. García (Eds.), Abriendo las puertas del aula. Transformación de las prácticas docentes (pp. 59-92). Universidad Católica de Chile.

Ferrada, D., Turra, O. y Villena, A. (2013). Currículum transformador de formación inicial para profesores en contextos de vulnerabilidad social. Cadernos de Pesquisa, 43(149), 642-661.

http://publicacoes.fcc.org.br/ojs/index.php/cp/article/view/2669/

Gariglio, J. A. y Burnier, S. L. (2014). Os professores da educação profissional: saberes e práticas [Los profesores de educación profesional: saberes y prácticas]. Cadernos de Pesquisa, 44(154), 934-959.

http://publicacoes.fcc.org.br/ojs/index.php/cp/article/view/2880

Glock, S. (2016). Stop talking out of turn: the influence of students' gender and ethnicity on preservice teachers' intervention strategies for student misbehavior. Teaching and Teacher Education, 56, 106-114. https://doi.org/10.1016/j.tate.2016.02.012

Hamilton, L. (2015). Early professional development in the scottish context: pre-service high school teachers and the management of behaviour in classrooms. Teacher Development, 19(3), 328-343.

https://doi.org/10.1080/13664530.2015.1032338

Harding, J. L. y Hbaci, I. (2015). Evaluating pre-service teachers math teaching experience from different perspectives. Universal Journal of Educational Research, 3(6), 382-389.

http://doi.org/10.13189/ujer.2015.030605

Hennessy, S., Rojas-Drummond, S., Highan, R., Márquez, A. M., Maine, F., Ríos, R. M., García-Carrión, R., Torreblanca, O. y Barrera, M. J. (2016). Developing a coding scheme for analysing classroom dialogue across educational contexts. Learning, Culture and Social Interaction, 9, 16-44.

https://doi.org/10.1016/j.lcsi.2015.12.001

Hepp, P. (2015). Desafíos de las políticas de integración de tecnologías en la formación inicial y continua de los docentes. En M. Poggi (Ed.), Mejorar los aprendizajes en la educación obligatoria: políticas y actores (pp. 195-214). Instituto Internacional de Planeamiento de la Educación-Unesco.

https://unesdoc.unesco.org/ark:/48223/pf0000234977

Macías, D. F. y Sánchez, J. A. (2015). Classroom management: a persistent challenge for pre-service foreign language teachers. PROFILE Issues in Teachers' Professional Development, 17(2), 81-99.

http://doi.org/10.15446/profile.v17n2.43641

Martinic, S. y Villalta, M. (2015). La gestión del tiempo en la sala de clases y los rendimientos escolares en escuelas con Jornada Escolar Completa en Chile. Perfiles Educativos, 37(147), 28-49.

https://doi.org/10.22201/iisue.24486167e.2015.147.47260

Martinic, S. y Villalta, M. (2016). Jornada escolar completa y organización del tiempo en la sala de clases de educación básica. En J. Manzi y M. R. García (Eds.), Abriendo las puertas del aula. Transformación de las prácticas docentes (pp. 317-348). Universidad Católica de Chile.

Martinic, S., Moreno, R., Müller, M., Pimentel, F., Rittershaussen, S., Calderón, M. y Cabezas, H. (2014). Análisis comparativo del componente de práctica en el currículo de formación profesional de médicos y profesores en la Pontificia Universidad Católica de Chile. Estudios Pedagógicos, 40(1), 179-196.

http://revistas.uach.cl/index.php/estped/article/view/2362

Martinic, S., Vergara, C. y Huepe, D. (2013). Uso del tiempo e interacciones en la sala de clases. Un estudio de caso en Chile. Revista Pro-Posições, 24(1), 123-135.

https://periodicos.sbu.unicamp.br/ojs/index.php/proposic/article/view/8642665 
Nathan, M. J. y Kim, S. (2009). Regulation of teacher elicitations in the mathematics classroom. Cognition and Instruction, 27(2), 91-120. http://doi.org/10.1080/07370000902797304

Pasmanik, D. y Cerón, R. (2005). Las prácticas pedagógicas en el aula como punto de partida para el análisis del proceso enseñanza-aprendizaje: un estudio de casos en la asignatura de Química. Estudios Pedagógicos, 31(2), 71-87. http://revistas.uach.cl/index.php/estped/article/view/3334

Pozo, J. I., Loo, C. y Martín, E. (2016). El cambio de las concepciones y las prácticas docentes como factor de cambio educativo. En J. Manzi y M. R. García (Eds.), Abriendo las puertas del aula. Transformaciones de las prácticas docentes (pp. 545-584). Universidad Católica de Chile.

Preiss, D., Calcagni, E., Espinoza, A. M. y Grau, V. (2016). ¿Cómo se enseña el lenguaje y las matemáticas en las salas de primer y segundo ciclo básico en Chile? Principales hallazgos de una serie de estudios observacionales en clases de lenguaje y matemáticas. En J. Manzi y M. R. García (Eds.), Abriendo las puertas del aula. Transformación de las prácticas docentes (pp. 153-184). Universidad Católica de Chile.

Saiz, I., Gorostegui, E. y Vilotta, D. (2014). Sobre la complejidad de la gestión en una clase de matemática: entre lo planificado y la realidad del aula. Modelización algebraica de problemas planteados en Z. Educación Matemática, 26(1), 41-72. http://somidem.com.mx/descargas/Vol26-1-2.pdf

Sánchez, G. I. y Jara, X. E. (2016). El contexto y los actores de la práctica: estudio sobre la representación del profesor en formación. Páginas de Educación, 9(2), 118-155. https://doi.org/10.22235/pe.v9i2.1296

Sinclair, J. M. y Coulthard, M. (1975). Toward an analysis of discourse: the English used by teachers and pupils. Oxford University Press.

Solís, M. C., Núñez, C., Contreras, I., Rittershaussen, S., Montecinos, C. y Walker, H. (2011). Condiciones de la formación práctica de los futuros profesores. Estudios Pedagógicos, 37(1), 127-147.

http://revistas.uach.cl/index.php/estped/article/view/2985

Steins, G., Wittrock, K. y Haep, A. (2015). Contents of classroom management: what is necessary, what is possible, how is it done at school? Creative Education, 6(19), 2045-2062.

https://doi.org/10.4236/ce.2015.619210

Stough, L. M., Montague, M. L., Landmark, L. J. y Williams-Diehm, K. (2015). Persistent classroom management training needs of experienced teachers. Journal of the Scholarship of Teaching and Learning, 15(5), 36. https://doi.org/10.14434/josotl.v15i5.13784

Vaillant, D. (2013). Formación inicial del profesorado en América Latina: dilemas centrales y perspectivas. Revista Española de Educación Comparada, 22, 185-206. https://doi.org/10.5944/reec.22.2013.9329

Veyrunes, P. y San Martín, J. S. (2016). Analizar las interacciones en el aula durante la clase dialogada: ¿Qué implicaciones existen para la formación de los profesores? En J. Manzi y M. R. García (Eds.), Abriendo las puertas del aula. Transformación de las prácticas docentes (pp. 93-124). Universidad Católica de Chile.

Villalta, M., Assael, C. y Martinic, S. (2013). Conocimiento escolar y procesos cognitivos en la interacción didáctica en la sala de clase. Perfiles Educativos, 35(141), 84-96.

https://doi.org/10.22201/iisue.24486167e.2013.141.40525 
Wells, G. y Mejía-Arauz, R. (2005). Toward dialogue in the classroom: learning and teaching through inquiry Working Papers on Culture, Education and Human Development, 1(4), 1-45.

http://hdl.handle.net/11117/3009 


\section{Anexo}

\section{CATEGORÍAS}

Tabla A. Fases de la clase

\begin{tabular}{l|l}
\hline Fases & Definición \\
\hline Pre-Inicio & $\begin{array}{l}\text { Se realizan actividades tales como: normar o disciplinar al grupo, pedir silencio, pasar } \\
\text { la lista, nombrar e identificar tareas asignadas. }\end{array}$ \\
\hline Inicio & $\begin{array}{l}\text { Introducción al tema de la clase, motivación vinculada con el contenido, } \\
\text { contextualización, recogida de antecedentes previos, recordar la materia pasada. } \\
\text { Se inicia con objetivos y preguntas centrados en el contenido de la clase, exposición } \\
\text { de contenidos; se entregan instrucciones relacionadas con el contenido o de una } \\
\text { actividad vinculada al tema de clase. } \\
\text { Resumir, sintetizar contenidos entregados, anunciar tareas o temas para la próxima } \\
\text { lección. } \\
\text { Finalización o cierre final social. Profesores y alumnos intercambian despedida; } \\
\text { anuncios sobre la próxima clase; en algunos casos se designan tareas para la clase. }\end{array}$ \\
\hline Cierre Pedagógico
\end{tabular}

Tabla B. Dirección del habla

\begin{tabular}{l|l}
\hline Dirección del habla & Definición \\
\hline A la Clase & Profesor se dirige a todos los participantes de la clase. \\
Al grupo & Profesor se dirige a un grupo de alumnos. \\
A un alumno & Profesor se dirige a un alumno específico. \\
Sin Intervención & No es evidente un destinatario específico del habla del profesor. \\
\hline
\end{tabular}

Tabla C. Intercambio

\begin{tabular}{|c|c|}
\hline Intercambio & Definición \\
\hline Expositivo & $\begin{array}{l}\text { El eje temático es la transmisión del contenido curricular (procedimental o conceptual). } \\
\text { Intercambio iniciado por el profesor. Estudiantes participan aceptando (de modo verbal o no } \\
\text { verbal) o repitiendo el contenido a solicitud del profesor y donde el cierre es la continuación de } \\
\text { transmisión de contenidos. }\end{array}$ \\
\hline Co-formado & $\begin{array}{l}\text { El eje temático es la evaluación de un contenido o procedimiento. El profesor inicia buscando por } \\
\text { parte del estudiante una respuesta predeterminada, ya sea eligiendo una alternativa o } \\
\text { produciendo una respuesta sobre conocimiento factual previamente entregado. El cierre es la } \\
\text { aprobación o reprobación de la intervención del estudiante. }\end{array}$ \\
\hline Regulativo & $\begin{array}{l}\text { El eje temático es regulación del orden de participación de estudiantes en la clase. Lo inicia el } \\
\text { profesor o el alumno. El cierre es la acción coherente con la intervención de inicio. }\end{array}$ \\
\hline Explicativo & $\begin{array}{l}\text { El eje temático es la comprensión de un contenido curricular. Lo inicia el estudiante quien solicita al } \\
\text { profesor información sobre el contenido curricular de la clase. El profesor utiliza dicha intervención } \\
\text { para repetir y agregar nueva información al contenido trabajado en clase. El cierre puede ser } \\
\text { explícito o tácito. }\end{array}$ \\
\hline Cooperativo & $\begin{array}{l}\text { El eje temático es la generación de nueva información por parte del estudiante para completar una } \\
\text { tarea previamente planteada (ejemplo: responder una guía, realizar una exposición). El profesor o } \\
\text { el estudiante inician solicitando o dando su respuesta a la tarea planteada. Las demás } \\
\text { intervenciones agregan nueva información a la intervención inicial. El cierre es la síntesis de } \\
\text { intervenciones anteriores o una aceptación breve de acuerdo tácito con dichas intervenciones. }\end{array}$ \\
\hline Colaborativo & $\begin{array}{l}\text { El eje es la reflexión o análisis que hace el estudiante de su propio actuar o intervención para } \\
\text { resolver o abordar un tema o problema que no tiene procesos ni respuestas predefinidas. El } \\
\text { profesor o el estudiante inician la indagación sobre las razones de una determinada intervención } \\
\text { anterior. Supone cierto desacuerdo entre los interlocutores que oriente la indagación recíproca. El } \\
\text { cierre es una aceptación recíproca de intervenciones. }\end{array}$ \\
\hline
\end{tabular}

Nota: Adaptado de Villalta et al., 2013. 
Tabla D. Intervención del profesor

\begin{tabular}{l|l}
\hline Intervención & Definición \\
\hline Exposición & $\begin{array}{l}\text { Profesor expone a la clase un contenido propio del tema o unidad definida. } \\
\text { Profesor realiza una pregunta, afirmación o petición dirigida a un alumno, a un grupo de } \\
\text { alumnos o al curso. Se realizan principalmente al inicio de un intercambio y obligan una } \\
\text { respuesta o acción en el alumno. } \\
\text { Ereguntas }\end{array}$ \\
$\begin{array}{l}\text { El profesor realiza acciones e instrucciones orientadas a disciplinar a alumnos y al grupo. } \\
\text { Ordena y regula la atención y el compromiso del estudiante con la tarea. } \\
\text { disciplinar } \\
\text { Regulación } \\
\text { de procedine reglas y normas para las actividades a realizar en relación con los contenidos } \\
\text { Responto } \\
\text { al alumno }\end{array}$ & $\begin{array}{l}\text { Responde a requerimiento del alumno respecto a contenidos de la clase, para evaluar } \\
\text { (positiva y/o negativamente), ejemplo: bien o mal (sin justificar por qué está bien o mal), o } \\
\text { para entregar retroalimentación; incorpora más contenidos o desarrolla una idea con } \\
\text { extensión. }\end{array}$ \\
\hline
\end{tabular}

